\title{
Large Deviations for Equilibrium Measures and Selection of Subaction
}

\author{
Jairo K. Mengue ${ }^{1}$
}

Received: 24 August 2016 / Accepted: 14 June 2017 / Published online: 23 June 2017

(C) Sociedade Brasileira de Matemática 2017

\begin{abstract}
Given a Lipschitz function $f:\{1, \ldots, d\}^{\mathbb{N}} \rightarrow \mathbb{R}$, for each $\beta>0$ we denote by $\mu_{\beta}$ the equilibrium measure of $\beta f$ and by $h_{\beta}$ the main eigenfunction of the Ruelle Operator $L_{\beta f}$. Assuming that $\left\{\mu_{\beta}\right\}_{\beta>0}$ satisfy a large deviation principle, we prove the existence of the uniform limit $V=\lim _{\beta \rightarrow+\infty} \frac{1}{\beta} \log \left(h_{\beta}\right)$. Furthermore, the expression of the deviation function is determined by its values at the points of the union of the supports of maximizing measures. We study a class of potentials having two ergodic maximizing measures and prove that a L.D.P. is satisfied. The deviation function is explicitly exhibited and does not coincide with the one that appears in the paper by Baraviera-Lopes-Thieullen which considers the case of potentials having a unique maximizing measure.
\end{abstract}

Keywords Equilibrium measure $\cdot$ Maximizing measure $\cdot$ Large deviation principle

\section{Introduction}

We denote by $X$ the Bernoulli space $\{1, \ldots, d\}^{\mathbb{N}}, \mathbb{N}=\{1,2,3, \ldots\}$, and by $\sigma$ the shift map acting on $X$. The metric considered satisfies $d_{\theta}(x, y)=\theta^{\min \left\{i, x_{i} \neq y_{i}\right\}}$, where $x=\left(x_{1} x_{2} x_{3} \cdots\right), y=\left(y_{1} y_{2} y_{3} \cdots\right)$ and $\theta \in(0,1)$ is fixed. If $x_{1}, \ldots, x_{n} \in$ $\{1, \ldots, d\}$ and $y=\left(y_{1} y_{2} y_{3} \cdots\right) \in X$, the notation $\left(x_{1} \cdots x_{n} y\right)$ represents the element $\left(x_{1} x_{2} \cdots x_{n} y_{1} y_{2} y_{3} \cdots\right) \in X$. A cylinder is a subset of $X$ of the form $\left[x_{1} \cdots x_{n}\right]:=\left\{\left(x_{1} \cdots x_{n} y\right) \mid y \in X\right\}$. We denote by $C(X)$ the set of continuous functions from $X$ to $\mathbb{R}$ and by $P(X)$ the set of probabilities on $X$.

\footnotetext{
Jairo K. Mengue

jairo.mengue@ufrgs.br

1 Universidade Federal do Rio Grande do Sul, Porto Alegre, Brazil
} 
Let $f: X \rightarrow \mathbb{R}$ be a Lipschitz function and, for each $\beta>0$, denote by $L_{\beta f}$ the Ruelle operator associated with $\beta f$, which is defined by

$$
L_{\beta f}: C(X) \rightarrow C(X), \quad\left(L_{\beta f}(w)\right)(x)=\sum_{a \in\{1, \ldots, d\}} e^{\beta f(a x)} w(a x) .
$$

We denote by $v_{\beta}$ the eigenmeasure of $L_{\beta f}$, that is, the probability satisfying $\int L_{\beta f}(u) d v_{\beta}=e^{P(\beta f)} \int u d v_{\beta}$ for any continuous function $u: X \rightarrow \mathbb{R}$, and by $h_{\beta}$ the main eigenfunction of $L_{\beta f}$. More precisely, $h_{\beta}$ is Lipschitz, $L_{\beta f}\left(h_{\beta}\right)=e^{P(\beta f)} h_{\beta}$ and $\int h_{\beta} d v_{\beta}=1$. Let $g_{\beta}:=\beta f+\log \left(h_{\beta}\right)-\log \left(h_{\beta} \circ \sigma\right)-P(\beta f)$. The functions $g_{\beta}$ and $\beta f-P(\beta f)$ are cohomologous and $L_{g_{\beta}} 1=1$. The eigenmeasure $\mu_{\beta}$ of $L_{g_{\beta}}$ is $\sigma$-invariant and coincides with the equilibrium measure of $\beta f$, that is,

$$
\int \beta f d \mu_{\beta}+h\left(\mu_{\beta}\right)=P(\beta f)=\sup _{\{\mu \in P(X) ; \mu \text { is } \sigma \text {-invariant }\}} \int \beta f d \mu+h(\mu) .
$$

Furthermore $d \mu_{\beta}=h_{\beta} d v_{\beta}$. Classical results on thermodynamic formalism can be found in Bowen et al. (2008) and Parry and Pollicott (1990).

At the zero temperature case, in thermodynamic formalism, the above objects are studied for large $\beta$. In this case some intersections with ergodic optimization appear (Baraviera et al. 2013; Contreras et al. 2001; Conze and Guivarc'h 1993; Jenkinson 2006). It is well known, for instance, that $\lim _{\beta \rightarrow+\infty} \frac{P(\beta f)}{\beta}=m(f)$, where

$$
m(f):=\sup _{\{\mu \in P(X) ; \mu \text { is } \sigma-\text { invariant }\}} \int f d \mu .
$$

Any possible limit (weak* topology) of a subsequence of $\left(\mu_{\beta}\right)_{\beta>0}$ attains the supremum in (1) that is, is a maximizing measure of $f$. Furthermore, the family of functions $\left(\frac{1}{\beta} \log \left(h_{\beta}\right)\right)$ is equicontinuous and uniformly bounded and any possible uniform limit $V$ of a subsequence of $\left(\frac{1}{\beta} \log \left(h_{\beta}\right)\right)$ is a calibrated subaction (Contreras et al. 2001), that is, it satisfies, for any $x \in X$, the equation

$$
\sup _{\sigma(y)=x}[f(y)+V(y)-V(x)-m(f)]=0 .
$$

The limit function $V$ is Lipschitz and $R_{-}:=f+V-V \circ \sigma-m(f)$ is the uniform limit of the corresponding subsequence of $\frac{g_{\beta}}{\beta}$, which satisfies:

(1) $R_{-}$is Lipschitz and $R_{-} \leq 0$,

(2) $R_{-}$and $f-m(f)$ are cohomologous,

(3) For any $x \in X$ there exists $y \in \sigma^{-1}(x)$ satisfying $R_{-}(y)=0$.

Define $R_{+}:=-R_{-}, R_{+}^{n}(x):=\sum_{j=0}^{n-1} R_{+}\left(\sigma^{j}(x)\right)$ and $R_{+}^{\infty}(x):=\lim _{n \rightarrow+\infty} R_{+}^{n}(x)$ $\left(R_{+}^{\infty}\right.$ can assume the value $\left.+\infty\right)$.

Subactions and maximizing measures are dual objects linked in a particular form when we study the speed of convergence of $\mu_{\beta}$ to a maximizing measure. From Baraviera et al. (2006) is known that, when the maximizing measure of $f$ is unique, 
there exists the uniform limit $R_{-}$of $\frac{g_{\beta}}{\beta},(\beta \rightarrow+\infty)$. Furthermore, the measures $\left(\mu_{\beta}\right)_{\beta>0}$ satisfy a large deviation principle (LDP), in the following sense, also used in the present work: there exists a lower semi-continuous function $I: X \rightarrow[0,+\infty]$ satisfying, for any cylinder $k \subset X$,

$$
\lim _{\beta \rightarrow+\infty} \frac{1}{\beta} \log \left(\mu_{\beta}(k)\right)=-\inf _{x \in k} I(x) .
$$

The deviation function $I$ in Baraviera et al. (2006) is given by $I=R_{+}^{\infty}$. It can assume the value $+\infty$.

In Lopes and Mengue (2014); Mengue (2010) this result has been generalized. Given $x \in X, n \in \mathbb{N}$ and $\beta>0$, consider the probability $m_{x, \beta, n} \in P(X)$ defined by $\int w d m_{x, \beta, n}=L_{g_{\beta}}^{n}(w)(x)$. If the maximizing measure of $f$ is unique, then ${ }^{1}$

$$
\lim _{n, \beta \rightarrow+\infty} \frac{1}{\beta} \log \left(m_{x, \beta, n}(k)\right)=\lim _{n, \beta \rightarrow+\infty} \frac{1}{\beta} \log \left(L_{g_{\beta}}^{n}\left(\chi_{k}\right)(x)\right)=-\inf _{z \in k} R_{+}^{\infty}(z)
$$

for any cylinder $k \subset X$. Given a continuous function $w, L_{g_{\beta}}^{n}(w)$ converges uniformly to $\int w d \mu_{\beta}(n \rightarrow+\infty)$. Therefore, for any $x \in X$, the probabilities $\mu_{x, \beta, n}$ converge to $\mu_{\beta}$ in the weak ${ }^{*}$ topology $(n \rightarrow+\infty)$. Consequently, from (2), for any $x \in X$,

$$
\lim _{\beta \rightarrow+\infty} \frac{1}{\beta} \log \left(\mu_{\beta}(k)\right)=\lim _{\beta \rightarrow+\infty} \lim _{n \rightarrow+\infty} \frac{1}{\beta} \log \left(m_{x, \beta, n}(k)\right)=-\inf _{z \in k} R_{+}^{\infty}(z) .
$$

In Bissacot et al. (2016b) the main result of Baraviera et al. (2006), stated above, was studied for a more general class of functions (satisfying the Walters condition) on a countable mixing subshift with the BIP property. However, in both works it was assumed the existence of a unique maximizing measure to $f$.

If we do not assume the hypothesis of unicity, then there are some natural questions to be considered:

Question 1: there exists $V:=\lim _{\beta \rightarrow+\infty} \frac{1}{\beta} \log \left(h_{\beta}\right)$ ?

Question 2: there exists $\lim _{\beta \rightarrow+\infty} \frac{1}{\beta} \log \left(\mu_{\beta}(k)\right)$ for any cylinder $k$ ?

Question 3: there are relations between $V$ and the deviation function $I$ ?

Initially, it is natural to assume that the answer to the question 3 can be obtained generalizing the results in Baraviera et al. (2006). In the case of existing the uniform limit $R_{+}=\lim _{\beta \rightarrow+\infty}-g_{\beta} / \beta$, we could try to prove that $\mu_{\beta}$ satisfies a LDP with deviation function $I=R_{+}^{\infty}$. However, in Baraviera et al. (2013) it is proved that this assertion is false. Even in the case there exist the limits in questions 1 and 2, one can get an explicit example where $I \neq R_{+}^{\infty}$.

1 we write $\lim _{n, \beta \rightarrow+\infty} a_{\beta, n}=a$, with $a \in \mathbb{R}$, if for any $\epsilon>0$ there exists $L>0$ such that $n, \beta>L \Rightarrow$ $\left|a_{\beta, n}-a\right|<\epsilon$.

In the Eq. (2), even though $R_{+}^{\infty}$ can assume the value $+\infty$, we have that $\inf _{z \in k} R_{+}^{\infty}(z)$ is finite because for any point $p$ that belongs to the support of the maximizing measure we have $R_{+}^{\infty}(p)=0$ and the set $\cup_{n \geq 1} \sigma^{-n}\{p\}$ is dense. 
We will show that, when the assertion in question 2 is satisfied, an affirmative answer to the question 1 exists. In this case, we also present an answer to the question 3 , determining relations between $R_{+}$and $I$. Several results are known concerning the problem of selection of a maximizing measure (Baraviera et al. 2013; Bissacot et al. 2016a; Brémont 2003; Chazottes and Hochman 2010; Coronel and RiveraLetelier 2015; Kempton 2011; Leplaideur 2005, 2012). In this work we present an improvement in the study of selection of the subaction as a consequence of our results on large deviations on the first part of the paper.

Define

$$
M_{\max }(f):=\left\{\mu \in P(X): \mu \text { is } \sigma-\text { invariant and } \int f d \mu=m(f)\right\}
$$

and

$$
X_{\max }(f):=\bigcup_{\mu \in M_{\max }(f)} \operatorname{supp}(\mu) .
$$

$X_{\text {max }}(f)$ is called the Mather set of $f$. It is compact, non-empty and for any invariant probability $v, \operatorname{supp}(v) \subset X_{\max }(f)$ iff $v$ is a maximizing measure of $f$ (Morris 2007, 2013).

In the Sect. 2. we will prove the following theorem:

Theorem 1 With the above notations, suppose that for any cylinder $k \subset X$, there exists $\lim _{\beta \rightarrow+\infty} \frac{1}{\beta} \log \left(\mu_{\beta}(k)\right)$. Then, denoting $x=\left(x_{1} x_{2} x_{3} \cdots\right)$,

1. The family of probabilities $\left(\mu_{\beta}\right)_{\beta>0}$ satisfies a LDP with deviation function $I: X \rightarrow[0,+\infty]$

$$
I(x):=-\lim _{n \rightarrow+\infty} \lim _{\beta \rightarrow+\infty} \frac{1}{\beta} \log \left(\mu_{\beta}\left(\left[x_{1} \cdots x_{n}\right]\right)\right) .
$$

2. There exists the uniform limit $R_{+}:=-\lim _{\beta \rightarrow+\infty} \frac{g_{\beta}}{\beta}$. It satisfies

$$
I=R_{+}+I \circ \sigma \quad \text { and } \quad I \geq R_{+}^{\infty} .
$$

3.

$$
I(x)=\inf _{y \in X_{\max }(f)} \liminf _{n \rightarrow+\infty}\left(R_{+}^{n}\left(x_{1} \cdots x_{n} y\right)+I(y)\right) .
$$

If $R_{+}^{\infty}(x)<+\infty$, there exists at least one point $y \in X_{\max }(f)$ which is an accumulation point of $\left\{\sigma^{n} x\right\}_{n=1,2, \ldots}$. For any such $y$

$$
I(x)=R_{+}^{\infty}(x)+I(y) .
$$

4. There exists the uniform limit $V=\lim _{\beta \rightarrow+\infty} \frac{1}{\beta} \log \left(h_{\beta}\right)$. 
5. The eigenmeasures $v_{\beta}$ satisfy a LDP with deviation function $I+V$.

Some remarks:

1. In the Eq. (3) we do not exclude the possibility $I(x)=+\infty$. When we write $I(x)=R_{+}(x)+I(\sigma x)$ we may have $+\infty=R_{+}(x)+\infty$. Following the above discussion, the function $R_{+}$is real-valued, nonnegative, Lipschitz and for any $x \in X, \min _{a \in\{1, \ldots, d\}} R_{+}(a x)=0$.

2. Under the hypothesis of the theorem we get:

2.1. There exists at least one point $\tilde{y} \in X_{\max }(f)$ satisfying $I(\tilde{y})=0$. Indeed, let $\mu_{\infty}$ be a probability on $X$ such that, for an increasing sequence $\beta_{i} \rightarrow+\infty$, $\mu_{\beta_{i}} \rightarrow \mu_{\infty}$ (weak* topology). Let $\tilde{y} \in \operatorname{supp}\left(\mu_{\infty}\right)$, that is, $\mu_{\infty}\left(\left[y_{1} \cdots y_{n}\right]\right)>0$ for any cylinder $\left[y_{1} \cdots y_{n}\right]$ containing $\tilde{y}$. In this way, from the hypothesis of the theorem,

$$
\lim _{\beta \rightarrow+\infty} \frac{1}{\beta} \log \left(\mu_{\beta}\left(\left[y_{1} \cdots y_{n}\right]\right)\right)=\lim _{\beta_{i} \rightarrow+\infty} \frac{1}{\beta_{i}} \log \left(\mu_{\beta_{i}}\left(\left[y_{1} \cdots y_{n}\right]\right)\right)=0,
$$

because $\mu_{\beta_{i}}\left(\left[y_{1} \cdots y_{n}\right]\right) \rightarrow \mu_{\infty}\left(\left[y_{1} \cdots y_{n}\right]\right)>0$. It follows from item 1. of the theorem that $I(\tilde{y})=0$.

2.2. If $R_{+}^{\infty}(x)<+\infty$ then $I(x)<+\infty$. Consequently, as $R_{+}^{\infty}(x)=0$ for any $x \in X_{\max }(f)$, we conclude that $I(x)<+\infty$ for any $x \in X_{\max }(f)$.

Indeed, in the proof of the Theorem 1 we will show that (see eq. (10) below)

$$
I(x) \leq \liminf _{n \rightarrow+\infty} R_{+}^{n}\left(x_{1} \cdots x_{n} y\right)+I(y) \quad \forall y \in X .
$$

As $I(\tilde{y})=0$ at some point $\tilde{y} \in X_{\max }(f)$ and $R_{+}$is Lipschitz, there exists a constant $c>0$ satisfying

$$
\begin{aligned}
& I(x) \leq \liminf _{n \rightarrow+\infty} R_{+}^{n}\left(x_{1} \cdots x_{n} \tilde{y}\right) \leq \liminf _{n \rightarrow+\infty} R_{+}^{n}(x)+c\left(\theta+\cdots+\theta^{n}\right) \\
& \quad \leq R_{+}^{\infty}(x)+\frac{c \theta}{1-\theta} .
\end{aligned}
$$

2.3. In the Eq. (4), if $R_{+}^{\infty}(x)=+\infty$ then, following computations as above, we get

$$
\liminf _{n \rightarrow+\infty}\left(R_{+}^{n}\left(x_{1} \cdots x_{n} y\right)+I(y)\right)=+\infty \forall y \in X_{\max }(f) .
$$

In this case we write

$$
\inf _{y \in X_{\max }(f)} \liminf _{n \rightarrow+\infty}\left(R_{+}^{n}\left(x_{1} \cdots x_{n} y\right)+I(y)\right)=+\infty .
$$

If $R_{+}^{\infty}(x)<+\infty$ then

$$
\liminf _{n \rightarrow+\infty}\left(R_{+}^{n}\left(x_{1} \cdots x_{n} y\right)+I(y)\right)<+\infty \forall y \in X_{\max }(f) .
$$


2.4. If $I(y)=0$ for all $y \in X_{\max }(f)$ then $I(x)=R_{+}^{\infty}(x)$ for all $x \in X$ (it follows from item 3. of the theorem). This is the case, for instance, if the maximizing measure of $f$ is unique, following the discussion in 2.1.

2.5. The Eq. (4) remains valid if we replace $y \in X_{\max }(f)$ by $y \in X$. It follows a similar argument with $\inf _{y \in X_{\max }(f)}$ replaced by $\inf _{y \in X}$ in the proof.

3. There exist constants $C_{1}, C_{2}>0$ satisfying, for any $x, y \in X, n \geq 1$, and $\beta$ sufficiently large,

$$
-\beta C_{1}<\log \left(h_{\beta}(x)\right)<\beta C_{1},\left|\log \left(h_{\beta}(x)\right)-\log \left(h_{\beta}(y)\right)\right|<\beta C_{1} d_{\theta}(x, y)
$$

and

$$
e^{-\beta n C_{2}}<\mu_{\beta}\left(\left[x_{1} \cdots x_{n}\right]\right)<e^{\beta n C_{2}} .
$$

For a proof of (5), see Contreras et al. (2001) p. 1404 or Mengue (2010) Lemma 28. For a proof of (6), see Parry and Pollicott (1990), proof of the Corollary 3.2.1., observing that $\mu_{\beta}$ is the equilibrium measure of $\beta f+\log \left(h_{\beta}\right)-\log \left(h_{\beta} \circ \sigma\right)-$ $P(\beta f)$, and use (5).

If the hypothesis of the theorem is not satisfied, from (6), applying a Cantor's diagonal argument, we obtain the existence of a sequence $\beta_{j}$ for which all limits $\lim _{\beta_{j} \rightarrow+\infty} \frac{1}{\beta_{j}} \log \left(\mu_{\beta_{j}}(k)\right)$ exist. A similar result is valid for this subsequence, with all $\beta$ replaced by $\beta_{j}$ in the statement of the theorem.

4. If $X$ is a subshift of finite type defined from an aperiodic matrix, the theorem remains valid except by the Eq. (4) which must be replaced by the following Eq.

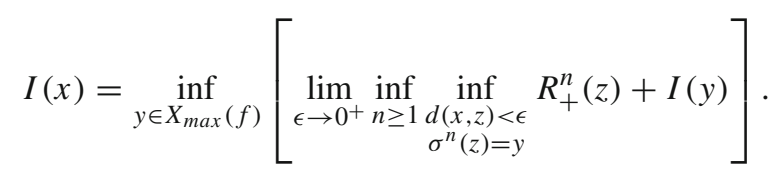

We will prove the Eq. (7) after the proof of the Theorem 1. Particullarly, both the Eqs. (4) and (7), are valid if $X=\{1, \ldots, d\}^{\mathbb{N}}$.

In the section 3. we will apply the above theorem studying the L.D.P. for the equilibrium measures of a class of Lipschitz functions $f:\{0,1\}^{\mathbb{N}} \rightarrow \mathbb{R}$ satisfying ${ }^{2}$

$$
\left.f\right|_{[01]}=b,\left.\quad f\right|_{[10]}=d, \quad f\left(0^{\infty}\right)=f\left(1^{\infty}\right)=0,\left.\quad f\right|_{\left[0^{n} 1\right]}=a_{n},\left.\quad f\right|_{\left[1^{n} 0\right]}=c_{n}, \quad n \geq 2
$$

where $b, d, a_{n}, c_{n}<0$. The deviation function $I$ is presented and, in the case $\sum_{j \geq 2} a_{j}<b+d+\sum_{j \geq 2} c_{j}$, this function differs from the one that appears in Baraviera et al. (2006) [see also Baraviera et al. (2013)].

2 we use the following notations

$$
0^{2}=00,0^{3}=000, \ldots, 0^{\infty}=(0000 \cdots), 1^{2}=11,1^{3}=111, \ldots, 1^{\infty}=(1111 \cdots) .
$$




\section{Proof of Theorem 1}

The following general result is very helpful and proves item 1 . of Theorem 1 .

Lemma 2 Let $\eta_{\beta}$ be a sequence of probabilities on $X$. Suppose that for any cylinder $k \subset X$ there exists the limit $\lim _{\beta \rightarrow+\infty} \frac{1}{\beta} \log \left(\eta_{\beta}(k)\right)$. Then, denoting $x=\left(x_{1} x_{2} x_{3} \cdots\right)$,

1. The function $I: X \rightarrow[0,+\infty]$,

$$
I(x):=-\lim _{n \rightarrow+\infty} \lim _{\beta \rightarrow+\infty} \frac{1}{\beta} \log \left(\eta_{\beta}\left(\left[x_{1} \cdots x_{n}\right]\right)\right)
$$

is lower semi-continuous.

2. For any cylinder $k \subset X$,

$$
\lim _{\beta \rightarrow+\infty} \frac{1}{\beta} \log \left(\eta_{\beta}(k)\right)=-\inf _{x \in k} I(x)
$$

Remark In Bissacot et al. (2016b) this result is generalized for Gibbs measures when considering a countable mixing subshift with the BIP property.

Proof The function $I$ is well defined because $\psi_{x}(n):=\lim _{\beta \rightarrow+\infty} \frac{1}{\beta} \log \left(\eta_{\beta}\left(\left[x_{1} \cdots\right.\right.\right.$ $\left.\left.\left.x_{n}\right]\right)\right)$ exists and it is not increasing with $n$. The function $I: X \rightarrow[0,+\infty], I(x)=$ $-\lim _{n \rightarrow+\infty} \psi_{x}(n)$ assume the value $+\infty$ if $\lim _{n \rightarrow+\infty} \psi_{x}(n)=-\infty$. Furthermore, denoting $z^{n}=\left(z_{1}^{n} z_{2}^{n} z_{3}^{n} \cdots\right) \in X$,

$$
\begin{aligned}
I(x) & =\liminf _{n \rightarrow+\infty}\left[-\lim _{\beta \rightarrow+\infty} \frac{1}{\beta} \log \left(\eta_{\beta}\left(\left[x_{1} \cdots x_{n}\right]\right)\right)\right] \\
& =\liminf _{n \rightarrow+\infty} \inf _{z^{n} \in\left[x_{1} \cdots x_{n}\right]}\left[-\lim _{m \rightarrow+\infty} \lim _{\beta \rightarrow+\infty} \frac{1}{\beta} \log \left(\eta_{\beta}\left(\left[x_{1} \cdots x_{n}\right]\right)\right)\right] \\
& \leq \liminf _{n \rightarrow+\infty} \inf _{z^{n} \in\left[x_{1} \cdots x_{n}\right]}\left[-\lim _{m \rightarrow+\infty} \lim _{\beta \rightarrow+\infty} \frac{1}{\beta} \log \left(\eta_{\beta}\left(\left[z_{1}^{n} \cdots z_{m}^{n}\right]\right)\right)\right] \\
& =\liminf _{n \rightarrow+\infty} \inf _{z^{n} \in\left[x_{1} \cdots x_{n}\right]} I\left(z^{n}\right),
\end{aligned}
$$

therefore $I$ is lower semi-continuous.

Given a cylinder $k=\left[x_{1} \cdots x_{n}\right]$, for any $z=\left(z_{1} z_{2} z_{3} \cdots\right) \in k$ we have

$$
\lim _{\beta \rightarrow+\infty} \frac{1}{\beta} \log \left(\eta_{\beta}(k)\right) \geq \lim _{m \rightarrow+\infty} \lim _{\beta \rightarrow+\infty} \frac{1}{\beta} \log \left(\eta_{\beta}\left(\left[z_{1} \cdots z_{m}\right]\right)\right)=-I(z) .
$$

Thus, we get

$$
\lim _{\beta \rightarrow+\infty} \frac{1}{\beta} \log \left(\eta_{\beta}(k)\right) \geq \sup _{z \in k}-I(z)=-\inf _{z \in k} I(z) .
$$


On the other hand, as

$$
\begin{aligned}
\lim _{\beta \rightarrow+\infty} \frac{1}{\beta} \log \left(\eta_{\beta}\left(\left[x_{1} \cdots x_{n}\right]\right)\right) & =\lim _{\beta \rightarrow+\infty} \frac{1}{\beta} \log \left(\sum_{j=1}^{d} \eta_{\beta}\left(\left[x_{1} \cdots x_{n} j\right]\right)\right) \\
& =\max _{j \in\{1, \cdots, d\}} \lim _{\beta \rightarrow+\infty} \frac{1}{\beta} \log \left(\eta_{\beta}\left(\left[x_{1} \cdots x_{n} j\right]\right)\right),
\end{aligned}
$$

there exists $y=\left(y_{1} y_{2} y_{3} \cdots\right) \in X$ satisfying

$$
\begin{aligned}
\lim _{\beta \rightarrow+\infty} \frac{1}{\beta} \log \left(\eta_{\beta}\left(\left[x_{1} \cdots x_{n}\right]\right)\right) & =\lim _{\beta \rightarrow+\infty} \frac{1}{\beta} \log \left(\eta_{\beta}\left(\left[x_{1} \cdots x_{n} y_{1}\right]\right)\right) \\
& =\lim _{\beta \rightarrow+\infty} \frac{1}{\beta} \log \left(\eta_{\beta}\left(\left[x_{1} \cdots x_{n} y_{1} y_{2}\right]\right)\right)=\cdots
\end{aligned}
$$

Therefore, we finally get

$$
\lim _{\beta \rightarrow+\infty} \frac{1}{\beta} \log \left(\eta_{\beta}(k)\right)=-I\left(x_{1} \cdots x_{n} y\right) \leq \sup _{z \in k}-I(z)=-\inf _{z \in k} I(z) .
$$

Lemma 3 Under the hypotheses of the Theorem 1 the deviation function I in (3) satisfies $I(x) \geq I(\sigma(x)) \forall x \in X$. Particularly, the function

$$
I_{0}: X \rightarrow[0,+\infty], \quad I_{0}(x):=\lim _{n \rightarrow+\infty} I\left(\sigma^{n}(x)\right)
$$

is constant on each orbit $\Omega_{x}=\left\{\sigma^{n}(x) \mid n \in\{0,1,2,3, \ldots\}\right\}, x \in X$.

Proof Denoting $x=\left(x_{1} x_{2} x_{3} \ldots\right)$, as $\mu_{\beta}$ is $\sigma$-invariant, for $n \geq 2$,

$$
\frac{1}{\beta} \log \left(\mu_{\beta}\left(\left[x_{1} \cdots x_{n}\right]\right)\right) \leq \frac{1}{\beta} \log \left(\sum_{j=1}^{d} \mu_{\beta}\left(\left[j x_{2} \cdots x_{n}\right]\right)\right)=\frac{1}{\beta} \log \left(\mu_{\beta}\left(\left[x_{2} \cdots x_{n}\right]\right)\right) .
$$

Then

$$
\begin{aligned}
I(x) & =-\lim _{n \rightarrow+\infty} \lim _{\beta \rightarrow+\infty} \frac{1}{\beta} \log \left(\mu_{\beta}\left(\left[x_{1} \cdots x_{n}\right]\right)\right) \\
& \geq-\lim _{n \rightarrow+\infty} \lim _{\beta \rightarrow+\infty} \frac{1}{\beta} \log \left(\mu_{\beta}\left(\left[x_{2} \cdots x_{n}\right]\right)\right)=I(\sigma(x)) .
\end{aligned}
$$

We write $y \in \omega(x), x, y \in X$, if there exists an increasing sequence $n_{i} \rightarrow+\infty$ such that $\sigma^{n_{i}}(x) \rightarrow y$.

Corollary 4 Under the hypotheses of Theorem 1, let I be the deviation function defined in (3) and $I_{0}$ be the function defined in (8).

1. If $y \in \omega(x)$, then $I(y) \leq I_{0}(x) \leq I(x)$, 
2. I is constant on each periodic orbit,

3. If $x \in \omega(y)$ and $y \in \omega(x)$, then $I_{0}(x)=I(x)=I(y)=I_{0}(y)$.

Proof In order to prove 1. we suppose $\sigma^{n_{j}}(x) \rightarrow y$. From the above lemma and using the lower semi-continuity of $I$ we get

$$
I(x) \geq I_{0}(x)=\lim _{n_{j} \rightarrow+\infty} I\left(\sigma^{n_{j}}(x)\right) \geq I(y) .
$$

The proof of 2. consists in observing that for a periodic orbit $\left\{x, \ldots, \sigma^{n}(x)\right\}$ we have:

$$
I(x) \geq I(\sigma(x)) \geq \cdots \geq I\left(\sigma^{n}(x)\right) \geq I(x) .
$$

Analogously, to prove 3. we observe that from 1. we have

$$
I(x) \geq I_{0}(x) \geq I(y) \geq I_{0}(y) \geq I(x) .
$$

\section{Proof of Theorem 1:}

proof of 1 .

It is a consequence of Lemma 2.

proof of 2 .

The existence of the limit $R_{-}$is a consequence of corollary 48 in Mengue (2010). Furthermore, following the Proposition 47 in Mengue (2010), for $x=\left(x_{0} x_{1} x_{2} \cdots\right)$, we get

$$
\begin{aligned}
R_{+}(x) & =\lim _{\beta, n \rightarrow+\infty} \frac{1}{\beta} \log \frac{\mu_{\beta}\left[x_{1} \cdots x_{n}\right]}{\mu_{\beta}\left[x_{0} \cdots x_{n}\right]} \\
& =\lim _{n \rightarrow+\infty}\left(\lim _{\beta \rightarrow+\infty} \frac{1}{\beta} \log \mu_{\beta}\left[x_{1} \cdots x_{n}\right]-\lim _{\beta \rightarrow+\infty} \frac{1}{\beta} \log \mu_{\beta}\left[x_{0} \cdots x_{n}\right]\right) .
\end{aligned}
$$

Therefore, using Lemma 2, we have

$$
I(x)=R_{+}(x)+I(\sigma(x)) .
$$

It follows that for each $n$,

$$
I(x)=R_{+}^{n}(x)+I\left(\sigma^{n}(x)\right),
$$

and, taking $n \rightarrow+\infty$,

$$
I(x)=R_{+}^{\infty}(x)+I_{0}(x)
$$

[see also Bissacot et al. (2016b)].

proof of 3 . 
Denoting $x=\left(x_{1} x_{2} x_{3} \cdots\right)$, we want to show that

$$
I(x)=\inf _{y \in X_{\max }(f)} \liminf _{n \rightarrow+\infty}\left(R_{+}^{n}\left(x_{1} \cdots x_{n} y\right)+I(y)\right) .
$$

For a fixed $y \in X$ we have from (9) that

$$
I\left(x_{1} \cdots x_{n} y\right)=R_{+}^{n}\left(x_{1} \cdots x_{n} y\right)+I(y) .
$$

As $I$ is lower semi-continuous,

$$
I(x) \leq \liminf _{n \rightarrow+\infty}\left(R_{+}^{n}\left(x_{1} \cdots x_{n} y\right)+I(y)\right) .
$$

Then, (considering an infimum on the right side)

$$
I(x) \leq \inf _{y \in X_{\max }(f)} \liminf _{n \rightarrow+\infty}\left(R_{+}^{n}\left(x_{1} \cdots x_{n} y\right)+I(y)\right) .
$$

Now, we will prove the reverse inequality.

If $R_{+}^{\infty}(x)=+\infty$, then $I(x)=R_{+}^{\infty}(x)+I_{0}(x)=+\infty$ and, as $R_{+}$is a Lipschitz function, for any $y \in X_{\max }(f)$,

$$
\liminf _{n \rightarrow+\infty} R_{+}^{n}\left(x_{1} \cdots x_{n} y\right)=+\infty
$$

So the main equality (4) holds.

If $R_{+}^{\infty}(x)<+\infty$, there exists at least one point $y \in X_{\max }(f)$ such that is an accumulation point of the sequence $\left\{\sigma^{n}(x)\right\}_{n=0,1, \ldots}$ [see Lopes et al. (2009) or Lemma 42 and Cor. 43 in Mengue (2010) or Bissacot et al. (2016b)]. We write $y=\left(y_{1} y_{2} y_{3} \cdots\right)$. It follows that for each $j \in \mathbb{N}$ there exists some $m_{j}>j$ such that

$$
x=\left(x_{1} \cdots x_{m_{j}} y_{1} \cdots y_{j} x_{m_{j}+j+1} x_{m_{j}+j+2} \cdots\right) .
$$

Then

$$
I(x)=\left(R_{+}^{m_{j}}\left(x_{1} \cdots x_{m_{j}} y_{1} \cdots y_{j} x_{m_{j}+j+1} \cdots\right)+I\left(y_{1} \cdots y_{j} x_{m_{j}+j+1} \cdots\right) .\right.
$$

When $j \rightarrow+\infty$, using the fact that $I$ is lower semi-continuous and $R_{+}$is Lipschitz, we get

$$
\begin{aligned}
I(x) & \geq \liminf _{j \rightarrow+\infty}\left(R_{+}^{m_{j}}\left(x_{1} \cdots x_{m_{j}} y_{1} \cdots y_{j} x_{m_{j}+j+1} \cdots\right)\right)+I(y) \\
& =\liminf _{j \rightarrow+\infty}\left(R_{+}^{m_{j}}\left(x_{1} \cdots x_{m_{j}} y\right)\right)+I(y) \\
& \geq \liminf _{n \rightarrow+\infty}\left(R_{+}^{n}\left(x_{1} \cdots x_{n} y\right)\right)+I(y) .
\end{aligned}
$$


Therefore,

$$
I(x) \geq \inf _{y \in X_{\max }(f)} \liminf _{n \rightarrow+\infty}\left(R_{+}^{n}\left(x_{1} \cdots x_{n} y\right)+I(y)\right),
$$

proving the reverse inequality. This concludes the proof of (4).

As we see above, if $R_{+}^{\infty}(x)<+\infty$, there exists at least one point $y \in X_{\max }(f)$ which is an accumulation point of $\left\{\sigma^{n} x\right\}_{n=1,2, \ldots}$. For any such $y$, from corollary 4 , we have

$$
I(x)=R_{+}^{\infty}(x)+I_{0}(x) \geq R_{+}^{\infty}(x)+I(y) .
$$

On the other hand, following the notations above

$$
\begin{aligned}
I(x) & \leq \liminf _{j \rightarrow+\infty} I\left(x_{1} \cdots x_{m_{j}} y\right) \\
& =\liminf _{j \rightarrow+\infty} R_{+}^{m_{j}}\left(x_{1} \cdots x_{m_{j}} y\right)+I(y)=\lim _{m_{j} \rightarrow+\infty} R_{+}^{m_{j}}(x)+I(y)=R_{+}^{\infty}(x)+I(y),
\end{aligned}
$$

where we use that $I$ is lower semi-continuous, $R_{+}^{n}(x)$ is increasing with $n$, $x_{m_{j}+1} \cdots x_{m_{j}+j}=y_{1} \cdots y_{j}$ and that $R_{+}$is Lipschitz. This concludes the proof of the equation

$$
I(x)=R_{+}^{\infty}(x)+I(y) .
$$

\section{Proof of 4. and 5.}

We denote by $v_{\beta}$ the eigenmeasure of the Ruelle Operator $L_{\beta f}$. The probabilities $v_{\beta}$ and $\mu_{\beta}$ satisfy $h_{\beta} d v_{\beta}=d \mu_{\beta}$, that is,

$$
\int w \cdot h_{\beta} d v_{\beta}=\int w d \mu_{\beta} \forall w \in C(X) .
$$

Consequently, given a cylinder $k \subset X$, from (5) and (6), there exists a constant $C_{k}$ such that, for $\beta$ sufficiently large, $-\beta C_{k}<\log \left(v_{\beta}(k)\right)<\beta C_{k}$.

We suppose initially the existence of the uniform limit

$$
V_{1}:=\lim _{\beta_{j} \rightarrow+\infty} \frac{1}{\beta_{j}} \log \left(h_{\beta_{j}}\right) .
$$

For a cylinder $k_{0}$ and an accumulation point $a$ of $\frac{1}{\beta_{j}} \log \left(v_{\beta_{j}}\left(k_{0}\right)\right)$, there exists a subsequence $\beta_{j_{i}}$ such that

$$
\lim _{\beta_{j_{i}} \rightarrow+\infty} \frac{1}{\beta_{j_{i}}} \log \left(v_{\beta_{j_{i}}}\left(k_{0}\right)\right)=a .
$$

Using a Cantor's diagonal argument we can suppose that for any cylinder $k$ there exists the limit of $\frac{1}{\beta_{j_{i}}} \log \left(v_{\beta_{j_{i}}}(k)\right)$. By hypothesis, $\mu_{\beta}$ satisfies a LDP with deviation function $I$. Fixed any point $z=\left(x_{1} x_{2} x_{3} \cdots\right)$, for each $n$, 


$$
\begin{gathered}
\frac{1}{\beta_{j_{i}}} \log \left(v_{\beta_{j_{i}}}\left(\left[x_{1} \cdots x_{n}\right]\right)+\inf _{\left[x_{1} \cdots x_{n}\right]} \frac{1}{\beta_{j_{i}}} \log \left(h_{\beta_{j_{i}}}\right) \leq \frac{1}{\beta_{j_{i}}} \log \left(\mu_{\beta_{j_{i}}}\left(\left[x_{1} \cdots x_{n}\right]\right)\right)\right. \\
\leq \frac{1}{\beta_{j_{i}}} \log \left(v_{\beta_{j_{i}}}\left(\left[x_{1} \cdots x_{n}\right]\right)+\sup _{\left[x_{1} \cdots x_{n}\right]} \frac{1}{\beta_{j_{i}}} \log \left(h_{\beta_{j_{i}}}\right) .\right.
\end{gathered}
$$

Taking $\beta_{j_{i}} \rightarrow+\infty$, we have

$$
\begin{gathered}
\lim _{\beta_{j_{i}} \rightarrow+\infty} \frac{1}{\beta_{j_{i}}} \log \left(v_{\beta_{j_{i}}}\left(\left[x_{1} \cdots x_{n}\right]\right)+\inf _{\left[x_{1} \cdots x_{n}\right]} V_{1} \leq \lim _{\beta_{j_{i}} \rightarrow+\infty} \frac{1}{\beta_{j_{i}}} \log \left(\mu_{\beta_{j_{i}}}\left(\left[x_{1} \cdots x_{n}\right]\right)\right)\right. \\
\leq \lim _{\beta_{j_{i}} \rightarrow+\infty} \frac{1}{\beta_{j_{i}}} \log \left(v_{\beta_{j_{i}}}\left(\left[x_{1} \cdots x_{n}\right]\right)+\sup _{\left[x_{1} \cdots x_{n}\right]} V_{1} .\right.
\end{gathered}
$$

When $n \rightarrow+\infty$ (applying Lemma 2) we have

$$
-\lim _{n \rightarrow+\infty} \lim _{\beta_{j_{i}} \rightarrow+\infty} \frac{1}{\beta_{j_{i}}} \log \left(v_{\beta_{j_{i}}}\left(\left[x_{1} \cdots x_{n}\right]\right)=I(z)+V_{1}(z) .\right.
$$

Using Lemma 2 again, we conclude that $v_{\beta_{j_{i}}}$ satisfies a LDP with deviation function $I+V_{1}$. Then

$$
a=-\inf _{x \in k_{0}}\left(I(x)+V_{1}(x)\right) .
$$

As $a$ is any possible accumulation point of $\frac{1}{\beta_{j}} \log \left(v_{\beta_{j}}\left(k_{0}\right)\right)$ we conclude that

$$
\lim _{\beta_{j} \rightarrow \infty} \frac{1}{\beta_{j}} \log \left(v_{\beta_{j}}\left(k_{0}\right)\right)=-\inf _{x \in k_{0}}\left(I(x)+V_{1}(x)\right) .
$$

Now we will prove the existence of the limit function $V$. Suppose that for subsequences $\beta_{i}$ and $\beta_{j}$ we have

$$
\lim _{\beta_{i} \rightarrow+\infty} \frac{1}{\beta_{i}} \log \left(h_{\beta_{i}}\right)=V_{1} \text { and } \lim _{\beta_{j} \rightarrow+\infty} \frac{1}{\beta_{j}} \log \left(h_{\beta_{j}}\right)=V_{2} .
$$

Applying 2. of the Theorem 1 we obtain $V_{2}-V_{2} \circ \sigma=V_{1}-V_{1} \circ \sigma$. Therefore, $V_{2}=V_{1}+C$ for some constant $C$.

Applying the above conclusions on the LDP for the set $X$ (the full space) we get

$$
0=\lim _{\beta_{i} \rightarrow+\infty} \frac{1}{\beta_{i}} \log \left(v_{\beta_{i}}(X)\right)=-\inf _{x \in X}\left(I(x)+V_{1}(x)\right)
$$

and

$$
0=\lim _{\beta_{j} \rightarrow+\infty} \frac{1}{\beta_{j}} \log \left(v_{\beta_{j}}(X)\right)=-\inf _{x \in X}\left(I(x)+V_{2}(x)\right) .
$$


Thus, we have

$$
\begin{aligned}
& 0=-\inf _{x \in X}\left(I(x)+V_{2}(x)\right)=-\inf _{x \in X}\left(I(x)+V_{1}(x)+C\right) \\
& =-\inf _{x \in X}\left(I(x)+V_{1}(x)\right)+C=0+C=C,
\end{aligned}
$$

proving that $V_{2}=V_{1}$. This shows that exists the uniform limit $V=\lim _{\beta \rightarrow \infty} \frac{1}{\beta} \log \left(h_{\beta}\right)$, proving 4.

The previous arguments on the LDP for the measures $v_{\beta_{j}}$ can be applied to the general family of measures $v_{\beta}$, proving 5 .

Proof of Remark 4 and Eq. (7): If $X$ is a subshift of finite type defined from an aperiodic matrix, the arguments used in the above proof are also valid except by some estimates in the proof of (4). In this case the Eq. (4) can be replaced by the Eq. (7), that is,

$$
I(x)=\inf _{y \in X_{\max }(f)}\left[\lim _{\epsilon \rightarrow 0^{+}} \inf _{n \geq 1} \inf _{\begin{array}{c}
(x, z)<\epsilon \\
\sigma^{n}(z)=y
\end{array}} R_{+}^{n}(z)+I(y)\right] .
$$

Indeed, from (9),

$$
\inf _{\substack{d(x, z)<\epsilon \\ \sigma^{n}(z)=y}} R_{+}^{n}(z)+I(y)=\inf _{\substack{d(x, z)<\epsilon \\ \sigma^{n}(z)=y}} I(z) .
$$

As $I$ is lower semi-continuous, for any $x, y \in X$, we have

$$
I(x) \leq\left[\lim _{\epsilon \rightarrow 0^{+}} \inf _{n \geq 1} \inf _{\substack{d(x, z)<\epsilon \\ \sigma^{n}(z)=y}} I(z)\right],
$$

and, then

$$
I(x) \leq \inf _{y \in X_{\max }(f)}\left[\lim _{\epsilon \rightarrow 0^{+}} \inf _{\substack{n \geq 1 \\ \inf _{d(x, z)<\epsilon} \\ \sigma^{n}(z)=y}} R_{+}^{n}(z)+I(y)\right]
$$

In order to prove the reverse inequality we remark that if $R_{+}^{\infty}(x)=+\infty$, then $I(x)=R_{+}^{\infty}(x)+I_{0}(x)=+\infty$ and using the above inequality, the Eq. (7) corresponds to the equality $+\infty=+\infty$. Suppose $R_{+}^{\infty}(x)<\infty$ and consider $\eta>0$. As $R_{+}$is Lipschitz, there exists a constant $C>0$ such that $\left|R_{+}(a)-R_{+}(b)\right| \leq$ $C d_{\theta}(a, b), \forall a, b \in X$. Let $j_{0}$ be such that $\frac{C \theta^{j_{0}}}{1-\theta}<\eta$. Take $y \in X_{\max }(f) \cap \omega(x)$. Then, from corollary 4 ,

$$
I(x)=R_{+}^{\infty}(x)+I_{0}(x) \geq R_{+}^{\infty}(x)+I(y) .
$$


Given $\epsilon>0$, let $j>j_{0}$ be such that $\theta^{j}<\epsilon$. For this $j$ there exists $m_{j}>j$ such that

$$
x=\left(x_{1} \cdots x_{m_{j}} y_{1} \cdots y_{j} x_{m_{j}+j+1} x_{m_{j}+j+2} \cdots\right) .
$$

Let $z_{\epsilon}=\left(x_{1} \cdots x_{m_{j}} y\right)$. Then,

$$
\begin{aligned}
I(x) & \geq R_{+}^{\infty}(x)+I(y) \geq R_{+}^{m_{j}}(x)+I(y) \\
& \geq R_{+}^{m_{j}}\left(x_{1} \cdots x_{m_{j}} y\right)+I(y)-\eta=R_{+}^{m_{j}}\left(z_{\epsilon}\right)+I(y)-\eta .
\end{aligned}
$$

Therefore, $d\left(x, z_{\epsilon}\right)<\epsilon, \sigma^{m_{j}}\left(z_{\epsilon}\right)=y$ and $I(x) \geq\left(R_{+}^{m_{j}}\left(z_{\epsilon}\right)+I(y)\right)-\eta$. This construction shows that

$$
I(x) \geq \inf _{y \in X_{\max }(f)}\left[\lim _{\epsilon \rightarrow 0} \inf _{\substack{n \geq 1 \\ \inf _{d(x, z)<\epsilon} \\ \sigma^{n}(z)=y}} R_{+}^{n}(z)+I(y)\right]-\eta .
$$

As $\eta$ can be arbitrarily small, we conclude the proof.

\section{Application for an Explicit Example}

Now we use the results described above in order to complete the study of Large Deviations for the equilibrium measures of a family of functions previously studied in Baraviera et al. (2013).

Definition 5 We write $f \in \mathbf{W}$ if $f:\{0,1\}^{\mathbb{N}} \rightarrow \mathbb{R}$ is a Lipschitz function and there exist negative numbers $b, d,\left\{c_{n}\right\}_{n \geq 2},\left\{a_{n}\right\}_{n \geq 2}$, such that, for $n \geq 2$,

$$
\left.f\right|_{[01]}=b,\left.\quad f\right|_{[10]}=d, \quad f\left(0^{\infty}\right)=f\left(1^{\infty}\right)=0,\left.\quad f\right|_{\left[0^{n} 1\right]}=a_{n},\left.\quad f\right|_{\left[1^{n} 0\right]}=c_{n} .
$$

Any function $f \in \mathbf{W}$ belongs to the class of potentials defined by P. Walters Walters (2007) where $0=a=c, b=b_{1}=b_{2}=\cdots$ and $d=d_{1}=d_{2}=\cdots$. We remark that $\sum_{i \geq 2} a_{i}>-\infty$ and $\sum_{i \geq 2} c_{i}>-\infty$, because $f$ is Lipschitz and $f\left(0^{\infty}\right)=f\left(1^{\infty}\right)=0$.

In the analysis of the zero temperature case for these functions, the exponential limit of $P(\beta f)$ plays an important role.

Lemma 6 If $f \in \mathbf{W}$ satisfies (11), then

$$
\lim _{\beta \rightarrow+\infty} \frac{1}{\beta} \log (P(\beta f))=A
$$

where

$$
A= \begin{cases}b+d+\sum_{j=1}^{\infty} c_{1+j}, & \text { when } \sum_{j=1}^{\infty} a_{1+j} \leq b+d+\sum_{j=1}^{\infty} c_{1+j}, \\ b+d+\sum_{j=1}^{\infty} a_{1+j}, & \text { when } \sum_{j=1}^{\infty} c_{1+j} \leq b+d+\sum_{j=1}^{\infty} a_{1+j}, \\ \frac{b+d}{2}+\sum_{j=1}^{\infty} \frac{a_{1+j}}{2}+\sum_{j=1}^{\infty} \frac{c_{1+j}}{2}, & \text { in the other cases. }\end{cases}
$$


Proof See Prop. 12 in Baraviera et al. (2013).

Given $f \in \mathbf{W}$ satisfying (11) and $\beta>0$, in order to simplify the computations, we will consider the function $H_{\beta}(x)=\frac{h_{\beta}(x)}{h_{\beta}\left(0^{\infty}\right)}$. This normalization of the eigenfunction was used in Baraviera et al. (2013). Observe that $H_{\beta}\left(0^{\infty}\right)=1$ and $\log \left(H_{\beta}\right)-\log \left(H_{\beta}\right.$ 。 $\sigma)=\log \left(h_{\beta}\right)-\log \left(h_{\beta} \circ \sigma\right)$. Therefore

$g_{\beta}=\beta f+\log \left(h_{\beta}\right)-\log \left(h_{\beta} \circ \sigma\right)-P(\beta f)=\beta f+\log \left(H_{\beta}\right)-\log \left(H_{\beta} \circ \sigma\right)-P(\beta f)$.

Following Walters (2007) (see Theo. 3.1 and p. 1341), we obtain

$$
\begin{aligned}
& H_{\beta}\left(0^{\infty}\right)=1 \\
& H_{\beta}\left(1^{\infty}\right)=\frac{e^{\beta b}}{e^{P(\beta f)}}\left(1+\sum_{j=1}^{\infty} e^{\beta\left(a_{2}+\cdots+a_{1+j}\right)-j P(\beta f)}\right) \\
& \left.H_{\beta}\right|_{\left[0^{q} 1\right]}=\frac{\left(e^{P(\beta f)}-1\right)}{e^{P(\beta f)}}\left(1+\sum_{j=1}^{\infty} e^{\beta\left(a_{q+1}+\cdots+a_{q+j}\right)-j P(\beta f)}\right), q \geq 1 \\
& \left.H_{\beta}\right|_{\left[1^{1} 0\right]}=\frac{H_{\beta}\left(1^{\infty}\right)\left(e^{P(\beta f)}-1\right)}{e^{P(\beta f)}}\left(1+\sum_{j=1}^{\infty} e^{\beta\left(c_{q+1}+\cdots+c_{q+j}\right)-j P(\beta f)}\right), q \geq 1 .
\end{aligned}
$$

The next lemma can be used in order to get the function $R_{+}^{\infty}$ that appears in the formulation of the deviation function in Theorem 1 .

Lemma 7 Under the above notations, for $f \in \mathbf{W}$ satisfying (11), there exists the uniform limit $U=\lim _{\beta \rightarrow+\infty} \frac{1}{\beta} \log \left(H_{\beta}\right)$. This function $U$ is a calibrated subaction for $f$ and satisfies

$U\left(0^{\infty}\right)=0$,

$U\left(1^{\infty}\right)=b+\max \left\{0, \sum_{j=1}^{\infty}\left(a_{1+j}\right)-A\right\}$

$\left.U\right|_{\left[0^{q} 1\right]}=A+\max \left\{0, \sum_{j=1}^{\infty}\left(a_{q+j}\right)-A\right\}, q \geq 1$,

$\left.U\right|_{\left[1^{q} 0\right]}=b+A+\max \left\{0, \sum_{j=1}^{\infty}\left(a_{1+j}\right)-A\right\}+\max \left\{0, \sum_{j=1}^{\infty}\left(c_{q+j}\right)-A\right\}, q \geq 1$.

Proof The result can be obtained as a particular case of Prop. 2 in Baraviera et al. (2013).

Remark if $V=\lim _{\beta \rightarrow+\infty} \frac{1}{\beta} \log \left(h_{\beta}\right)$ then $U=V-C$, with $C=V\left(0^{\infty}\right)$. 
We want to study the LDP for the equilibrium measures $\mu_{\beta}$ and naturally any maximizing measure of $f \in \mathbf{W}$ is a convex combination of the ergodic measures supported in the periodic orbits $0^{\infty}=(000 \ldots)$ and $1^{\infty}=(111 \ldots)$. From the above lemma there exists the limit function

$$
R_{+}=-\lim _{\beta \rightarrow+\infty} \frac{g_{\beta}}{\beta}=-f-U+U \circ \sigma+m(f)=-f-U+U \circ \sigma .
$$

We want to use Theorem 1. In order to do that we need to find the expression of deviation function. More precisely, we need to compute $I\left(0^{\infty}\right)$ and $I\left(1^{\infty}\right)$. In this way, considering the Lemma 2 , we first study $\mu_{\beta}\left(\left[0^{n}\right]\right)$ and $\mu_{\beta}\left(\left[1^{n}\right]\right)$.

Lemma 8 Let $f \in \mathbf{W}$ satisfying (11). Then, for $\beta>0$ and $n \geq 1$,

$$
\mu_{\beta}\left(\left[0^{n}\right]\right)=\frac{S_{0}^{n}(\beta)}{S_{0}(\beta)+S_{1}(\beta)} \text { and } \mu_{\beta}\left(\left[1^{n}\right]\right)=\frac{S_{1}^{n}(\beta)}{S_{0}(\beta)+S_{1}(\beta)} \text {, }
$$

where

$$
\begin{aligned}
& S_{0}(\beta)=S_{0}^{1}(\beta):=\frac{1+\sum_{j=1}^{\infty}(j+1) e^{\beta\left(a_{2}+\cdots+a_{1+j}\right)-j P(\beta f)}}{1+\sum_{j=1}^{\infty} e^{\beta\left(a_{2}+\cdots+a_{1+j}\right)-j P(\beta f)}}, \\
& S_{1}(\beta)=S_{1}^{1}(\beta):=\frac{1+\sum_{j=1}^{\infty}(j+1) e^{\beta\left(c_{2}+\cdots+c_{1+j}\right)-j P(\beta f)}}{1+\sum_{j=1}^{\infty} e^{\beta\left(c_{2}+\cdots+c_{1+j}\right)-j P(\beta f)}},
\end{aligned}
$$

and for $n \geq 2$

$$
\begin{aligned}
S_{0}^{n}(\beta) & :=\frac{e^{P(\beta f)} \sum_{j=n}^{\infty}(j-n+1) e^{\beta\left(a_{2}+\cdots+a_{j}\right)-j P(\beta f)}}{\left(1+\sum_{i=1}^{\infty} e^{\beta\left(a_{2}+\cdots+a_{1+i}\right)-i P(\beta f)}\right)}, \\
S_{1}^{n}(\beta) & :=\frac{e^{P(\beta f)} \sum_{j=n}^{\infty}(j-n+1) e^{\beta\left(c_{2}+\cdots+c_{j}\right)-j P(\beta f)}}{\left(1+\sum_{i=1}^{\infty} e^{\beta\left(c_{2}+\cdots+c_{1+i}\right)-i P(\beta f)}\right)} .
\end{aligned}
$$

Proof Following Baraviera et al. (2013), page 1351,

$$
\begin{aligned}
S_{0}(\beta) & :=\frac{1+\sum_{j=1}^{\infty}(j+1) e^{\beta\left(a_{2}+\cdots+a_{1+j}\right)-j P(\beta f)}}{1+\sum_{j=1}^{\infty} e^{\beta\left(a_{2}+\cdots+a_{1+j}\right)-j P(\beta f)}} \\
& =1+\sum_{j=2}^{\infty} e^{\beta\left(a_{2}+\cdots+a_{j}\right)+\log \left(\left.H_{\beta}\right|_{\left[0_{1} j_{1}\right.}\right)-\log \left(\left.H_{\beta}\right|_{[01]}\right)-(j-1) P(\beta f)}
\end{aligned}
$$

and

$$
S_{1}(\beta):=\frac{1+\sum_{j=1}^{\infty}(j+1) e^{\beta\left(c_{2}+\cdots+c_{1+j}\right)-j P(\beta f)}}{1+\sum_{j=1}^{\infty} e^{\beta\left(c_{2}+\cdots+c_{1+j}\right)-j P(\beta f)}}
$$




$$
=1+\sum_{j=2}^{\infty} e^{\beta\left(c_{2}+\cdots+c_{j}\right)+\log \left(\left.H_{\beta}\right|_{\left[1{ }^{j} 0\right]}\right)-\log \left(\left.H_{\beta}\right|_{[10]}\right)-(j-1) P(\beta f)} .
$$

For $j \geq 2$, [see p. 1352 in Baraviera et al. (2013)]

$$
\mu_{\beta}\left(\left[0^{j} 1\right]\right)=\mu_{\beta}([01]) e^{\beta\left(a_{2}+\cdots+a_{j}\right)+\log \left(\left.H_{\beta}\right|_{\left[0{ }^{j} 1\right]}\right)-\log \left(\left.H_{\beta}\right|_{[01]}\right)-(j-1) P(\beta f)}
$$

and

$$
\mu_{\beta}\left(\left[1^{j} 0\right]\right)=\mu_{\beta}([10]) e^{\beta\left(c_{2}+\cdots+c_{j}\right)+\log \left(\left.H_{\beta}\right|_{\left[1 j_{0]}\right.}\right)-\log \left(\left.H_{\beta}\right|_{[10]}\right)-(j-1) P(\beta f)} .
$$

Then

$$
\mu_{\beta}([0])=\sum_{j=1}^{\infty} \mu_{\beta}\left(\left[0^{j} 1\right]\right)=\mu_{\beta}([01]) S_{0}(\beta)
$$

and

$$
\mu_{\beta}([1])=\sum_{j=1}^{\infty} \mu_{\beta}\left(\left[1^{j} 0\right]\right)=\mu_{\beta}([10]) S_{1}(\beta)
$$

As $\mu_{\beta}([01])=\mu_{\beta}([10])$ (because $\mu_{\beta}$ is $\sigma$-invariant) and $\mu_{\beta}([0])+\mu_{\beta}([1])=1$ we obtain

$$
\mu_{\beta}([01])=\mu_{\beta}([10])=\frac{1}{S_{0}(\beta)+S_{1}(\beta)} .
$$

As a consequence,

$$
\mu_{\beta}([0])=\frac{S_{0}(\beta)}{S_{0}(\beta)+S_{1}(\beta)} \text { and } \mu_{\beta}([1])=\frac{S_{1}(\beta)}{S_{0}(\beta)+S_{1}(\beta)} \text {. }
$$

From (13) and (15), for any $n \geq 2$,

$$
\begin{aligned}
\mu_{\beta}\left(\left[0^{n}\right]\right) & =\sum_{j=n}^{\infty} \mu_{\beta}\left(\left[0^{j} 1\right]\right) \\
& =\mu_{\beta}([01]) \sum_{j=n}^{\infty} e^{\beta\left(a_{2}+\cdots+a_{j}\right)+\log \left(\left.H_{\beta}\right|_{\left[0^{j}{ }_{1}\right.}\right)-\log \left(\left.H_{\beta}\right|_{[01]}\right)-(j-1) P(\beta f)} \\
& =\frac{\sum_{j=n}^{\infty} e^{\beta\left(a_{2}+\cdots+a_{j}\right)+\log \left(\left.H_{\beta}\right|_{\left[0^{j}{ }_{1}\right.}\right)-\log \left(\left.H_{\beta}\right|_{[01]}\right)-(j-1) P(\beta f)}}{S_{0}(\beta)+S_{1}(\beta)}
\end{aligned}
$$


Furthermore,

$$
\begin{aligned}
\sum_{j=n}^{\infty} e^{\beta\left(a_{2}+\cdots+a_{j}\right)+\log \left(\left.H_{\beta}\right|_{\left[0^{j} 1\right]}\right)-\log \left(\left.H_{\beta}\right|_{[01]}\right)-(j-1) P(\beta f)} \\
\quad=\sum_{j=n}^{\infty} \frac{\left.e^{\beta\left(a_{2}+\cdots+a_{j}\right)-(j-1) P(\beta f)} H_{\beta}\right|_{\left[0^{j} 1\right]}}{\left.H_{\beta}\right|_{[01]}} \\
=\sum_{j=n}^{\infty} \frac{e^{\beta\left(a_{2}+\cdots+a_{j}\right)-(j-1) P(\beta f)}\left(1+\sum_{i=1}^{\infty} e^{\beta\left(a_{j+1}+\cdots+a_{j+i}\right)-i P(\beta f)}\right)}{\left(1+\sum_{i=1}^{\infty} e^{\beta\left(a_{2}+\cdots+a_{1+i}\right)-i P(\beta f)}\right)} \\
=\frac{\sum_{j=n}^{\infty} \sum_{i=0}^{\infty} e^{\beta\left(a_{2}+\cdots+a_{j+i}\right)-(j+i-1) P(\beta f)}}{\left(1+\sum_{i=1}^{\infty} e^{\beta\left(a_{2}+\cdots+a_{1+i}\right)-i P(\beta f)}\right)} \\
=\frac{e^{P(\beta f)} \sum_{m=n}^{\infty}(m-n+1) e^{\beta\left(a_{2}+\cdots+a_{m}\right)-m P(\beta f)}}{\left(1+\sum_{i=1}^{\infty} e^{\beta\left(a_{2}+\cdots+a_{1+i}\right)-i P(\beta f)}\right)}=S_{0}^{n}(\beta) .
\end{aligned}
$$

Therefore, we finally get

$$
\mu_{\beta}\left[0^{n}\right]=\frac{S_{0}^{n}(\beta)}{S_{0}(\beta)+S_{1}(\beta)} .
$$

The computation for $\mu_{\beta}\left[1^{n}\right]$ is similar.

As we want to determine the limit of $\frac{1}{\beta} \log \left(\mu_{\beta}\left(\left[0^{n}\right]\right)\right)$ and $\frac{1}{\beta} \log \left(\mu_{\beta}\left(\left[1^{n}\right]\right)\right)$ (see Lemma 2) the next lemma is useful.

Lemma 9 Let $f \in \mathbf{W}$ satisfying (11). Denote $A=\lim _{\beta \rightarrow+\infty} \frac{1}{\beta} \log (P(\beta f))$. Under the above notations,

$$
\begin{aligned}
& \lim _{\beta \rightarrow+\infty} \frac{1}{\beta} \log \left(S_{0}(\beta)\right)=\max \left\{0, \sum_{j=2}^{\infty} a_{j}-2 A\right\}-\max \left\{0, \sum_{j=2}^{\infty} a_{j}-A\right\}, \\
& \lim _{\beta \rightarrow+\infty} \frac{1}{\beta} \log \left(S_{1}(\beta)\right)=\max \left\{0, \sum_{j=2}^{\infty} c_{j}-2 A\right\}-\max \left\{0, \sum_{j=2}^{\infty} c_{j}-A\right\}
\end{aligned}
$$

and for $n \geq 2$

$$
\begin{aligned}
& \lim _{\beta \rightarrow+\infty} \frac{1}{\beta} \log \left(S_{0}^{n}(\beta)\right)=\max \left\{a_{2}+\cdots+a_{n}, \sum_{j=2}^{\infty} a_{j}-2 A\right\}-\max \left\{0, \sum_{j=2}^{\infty} a_{j}-A\right\}, \\
& \lim _{\beta \rightarrow+\infty} \frac{1}{\beta} \log \left(S_{1}^{n}(\beta)\right)=\max \left\{c_{2}+\cdots+c_{n}, \sum_{j=2}^{\infty} c_{j}-2 A\right\}-\max \left\{0, \sum_{j=2}^{\infty} c_{j}-A\right\} .
\end{aligned}
$$


Proof We only present the prove of the first equation, because the arguments are similar for the other cases. Initially, observe that for any $j_{1} \geq 0$,

$$
\lim _{\beta \rightarrow+\infty} \frac{1}{\beta} \log \left(\sum_{j \geq j_{1}}(j+1) e^{-j P(\beta f)}\right)=-2 A
$$

and

$$
\lim _{\beta \rightarrow+\infty} \frac{1}{\beta} \log \left(\sum_{j \geq j_{1}} e^{-j P(\beta f)}\right)=-A
$$

(see Cor. 14 in Baraviera et al. (2013)).

As

$$
\begin{aligned}
\frac{1}{\beta} \log \left(S_{0}(\beta)\right)= & \frac{1}{\beta} \log \left[1+\sum_{j=1}^{\infty}(j+1) e^{\beta\left(a_{2}+\cdots+a_{1+j}\right)-j P(\beta f)}\right] \\
& -\frac{1}{\beta} \log \left[1+\sum_{j=1}^{\infty} e^{\beta\left(a_{2}+\cdots+a_{1+j}\right)-j P(\beta f)}\right]
\end{aligned}
$$

we will study the limit for $\frac{1}{\beta} \log \left[1+\sum_{j=1}^{\infty}(j+1) e^{\beta\left(a_{2}+\cdots+a_{1+j}\right)-j P(\beta f)}\right]$ and $\frac{1}{\beta} \log [1+$ $\left.\sum_{j=1}^{\infty} e^{\beta\left(a_{2}+\cdots+a_{1+j}\right)-j P(\beta f)}\right]$.

As $a_{i}<0 \forall i \in\{2,3,4, \ldots\}$ we have,

$$
\begin{aligned}
\liminf _{\beta \rightarrow+\infty} & \frac{1}{\beta} \log \left(1+\sum_{j=1}^{\infty}(j+1) e^{\beta\left(a_{2}+\cdots+a_{1+j}\right)-j P(\beta f)}\right) \\
& \geq \max \left\{0, \liminf _{\beta \rightarrow+\infty} \frac{1}{\beta} \log \left(e^{\beta \sum_{i \geq 2} a_{i}} \sum_{j=1}^{\infty}(j+1) e^{-j P(\beta f)}\right)\right\} \\
& =\max \left\{0, \sum_{i \geq 2} a_{i}-2 A\right\} .
\end{aligned}
$$

Furthermore, for any fixed $j_{0}$, rewriting

$$
\left(1+\sum_{j=1}^{\infty}(j+1) e^{\beta\left(a_{2}+\cdots+a_{1+j}\right)-j P(\beta f)}\right)
$$

in the form

$$
\left[1+\sum_{j=1}^{j_{0}-1}(j+1) e^{\beta\left(a_{2}+\cdots+a_{1+j}\right)-j P(\beta f)}\right]+\left[\sum_{j=j_{0}}^{\infty}(j+1) e^{\beta\left(a_{2}+\cdots+a_{1+j}\right)-j P(\beta f)}\right]
$$


we have

$$
\begin{aligned}
& \limsup _{\beta \rightarrow+\infty} \frac{1}{\beta} \log \left(1+\sum_{j=1}^{\infty}(j+1) e^{\beta\left(a_{2}+\cdots+a_{1+j}\right)-j P(\beta f)}\right) \\
& \quad=\max \left\{0, \limsup _{\beta \rightarrow+\infty} \frac{1}{\beta} \log \left(\sum_{j=j_{0}}^{\infty}(j+1) e^{\beta\left(a_{2}+\cdots+a_{1+j}\right)-j P(\beta f)}\right)\right\} \\
& \leq \max \left\{0, \limsup _{\beta \rightarrow+\infty} \frac{1}{\beta} \log \left(e^{\beta\left(a_{2}+\cdots+a_{j_{0}}\right)} \sum_{j=j_{0}}^{\infty}(j+1) e^{-j P(\beta f)}\right)\right\} \\
& \quad=\max \left\{0, a_{2}+\ldots+a_{j_{0}}-2 A\right\} .
\end{aligned}
$$

Thus, as we can consider $j_{0}$ large enough,

$$
\limsup _{\beta \rightarrow+\infty} \frac{1}{\beta} \log \left(1+\sum_{j=1}^{\infty}(j+1) e^{\beta\left(a_{2}+\cdots+a_{1+j}\right)-j P(\beta f)}\right) \leq \max \left\{0, \sum_{j=2}^{\infty} a_{j}-2 A\right\}
$$

The conclusion is that

$$
\lim _{\beta \rightarrow+\infty} \frac{1}{\beta} \log \left(1+\sum_{j=1}^{\infty}(j+1) e^{\beta\left(a_{2}+\cdots+a_{1+j}\right)-j P(\beta f)}\right)=\max \left\{0, \sum_{j=2}^{\infty} a_{j}-2 A\right\} .
$$

With similar arguments we obtain

$$
\lim _{\beta \rightarrow+\infty} \frac{1}{\beta} \log \left(1+\sum_{j=1}^{\infty} e^{\beta\left(a_{2}+\cdots+a_{1+j}\right)-j P(\beta f)}\right)=\max \left\{0, \sum_{j=2}^{\infty} a_{j}-A\right\} .
$$

Now we show that the family of measures $\mu_{\beta}$ satisfies a L.D.P. and present the expression of the deviation function. We remark that, when the maximizing measure of a potential $f$ is unique, the deviation function in Baraviera et al. (2006) is equal to $R_{+}^{\infty}$. For the class of potentials that we consider in this section, we have $R_{+}^{\infty}\left(0^{\infty}\right)=$ $R_{+}^{\infty}\left(1^{\infty}\right)=0$. However in the theorem below $I\left(0^{\infty}\right) \neq 0$, which means, $I \neq R_{+}^{\infty}$ (see also Theo. 3 and p. 1343 in Baraviera et al. (2013)).

Theorem 10 Let $f \in \mathbf{W}$ satisfying (11). Suppose $\sum_{j \geq 2} a_{j}<b+d+\sum_{j \geq 2} c_{j}$. Then $\left(\mu_{\beta}\right)_{\beta>0}$ satisfies a Large Deviation Principle with deviation function I defined by

$$
I\left(0^{\infty}\right)=b+d+\sum_{j \geq 2} c_{j}-\sum_{j \geq 2} a_{j}, \quad I\left(1^{\infty}\right)=0
$$


and for any $x \in\{0,1\}^{\mathbb{N}}$,

$$
I(x)= \begin{cases}R_{+}^{n}(x)+I\left(0^{\infty}\right) & \text { if } x=\left(x_{1} \cdots x_{n} 0^{\infty}\right) \\ R_{+}^{n}(x) & \text { if } x=\left(x_{1} \cdots x_{n} 1^{\infty}\right) \\ +\infty & \text { else }\end{cases}
$$

where $R_{+}=-f-U+U \circ \sigma$ and $U$ satisfies

$$
\begin{gathered}
U\left(0^{\infty}\right)=0,\left.\quad U\right|_{\left[0^{q} 1\right]}=\max \left\{b+d+\sum_{j=2}^{\infty} c_{j}, \sum_{j=1}^{\infty} a_{q+j}\right\}, q \geq 1, \\
U\left(1^{\infty}\right)=b, \quad \text { and }\left.\quad U\right|_{\left[1^{q} 0\right]}=b+\sum_{j=1}^{\infty} c_{q+j}, q \geq 1 .
\end{gathered}
$$

Proof First note that, with the hypothesis $\sum_{j \geq 2} a_{j}<b+d+\sum_{j \geq 2} c_{j}$, from Lemma $6, A:=\lim _{\beta \rightarrow+\infty} \frac{1}{\beta} \log (P(\beta f))=b+d+\sum_{j \geq 2} c_{j}$. Then,

$$
\sum_{j \geq 2} a_{j}<A<\sum_{j \geq 2} c_{j}
$$

and this function $U$ coincides with the one in Lemma 7, which means, $U=$ $\lim _{\beta \rightarrow+\infty} \frac{1}{\beta} \log \left(H_{\beta}\right)$. Particularly, $R_{+}=-f-U+U \circ \sigma$ is the uniform limit of $-\frac{g_{\beta}}{\beta}$, when $\beta \rightarrow+\infty$.

claim:

$$
\lim _{n \rightarrow+\infty} \lim _{\beta \rightarrow+\infty} \frac{1}{\beta} \log \left(\mu_{\beta}\left(\left[0^{n}\right]\right)\right)=\sum_{j \geq 2} a_{j}-A
$$

and

$$
\lim _{n \rightarrow+\infty} \lim _{\beta \rightarrow+\infty} \frac{1}{\beta} \log \left(\mu_{\beta}\left(\left[1^{n}\right]\right)\right)=0
$$

Indeed, from (16), Lemma 8 and Lemma 9 we get, for $\mathrm{n}$ large enough,

$$
\begin{aligned}
\lim _{\beta \rightarrow+\infty} & \frac{1}{\beta} \log \left(\mu_{\beta}\left(\left[0^{n}\right]\right)\right)=\lim _{\beta \rightarrow+\infty} \frac{1}{\beta} \log \left(\frac{S_{0}^{n}(\beta)}{S_{0}(\beta)+S_{1}(\beta)}\right) \\
& =\lim _{\beta \rightarrow+\infty} \frac{1}{\beta} \log \left(S_{0}^{n}(\beta)\right)-\max \left\{\lim _{\beta \rightarrow+\infty} \frac{1}{\beta} \log \left(S_{0}(\beta)\right), \lim _{\beta \rightarrow+\infty} \frac{1}{\beta} \log \left(S_{1}(\beta)\right)\right\} \\
& =\left[\sum_{j=2}^{\infty} a_{j}-2 A\right]-\max \left\{\max \left\{0, \sum_{j=2}^{\infty} a_{j}-2 A\right\},-A\right\}
\end{aligned}
$$




$$
=\left[\sum_{j=2}^{\infty} a_{j}-2 A\right]-(-A)=\sum_{j=2}^{\infty} a_{j}-A
$$

and

$$
\begin{aligned}
\lim _{\beta \rightarrow+\infty} & \frac{1}{\beta} \log \left(\mu_{\beta}\left(\left[1^{n}\right]\right)\right)=\lim _{\beta \rightarrow+\infty} \frac{1}{\beta} \log \left(\frac{S_{1}^{n}(\beta)}{S_{0}(\beta)+S_{1}(\beta)}\right) \\
& =\lim _{\beta \rightarrow+\infty} \frac{1}{\beta} \log \left(S_{1}^{n}(\beta)\right)-\max \left\{\lim _{\beta \rightarrow+\infty} \frac{1}{\beta} \log \left(S_{0}(\beta)\right), \lim _{\beta \rightarrow+\infty} \frac{1}{\beta} \log \left(S_{1}(\beta)\right)\right\} \\
& =(-A)-(-A)=0 .
\end{aligned}
$$

This concludes the proof of claim.

Let $I: X \rightarrow[0,+\infty]$ be defined by

$$
\begin{aligned}
& I\left(0^{\infty}\right)=-\lim _{n \rightarrow+\infty} \lim _{\beta \rightarrow+\infty} \frac{1}{\beta} \log \left(\mu_{\beta}\left(\left[0^{n}\right]\right)\right)=b+d+\sum_{j \geq 2} c_{j}-\sum_{j \geq 2} a_{j}, \\
& I\left(1^{\infty}\right)=-\lim _{n \rightarrow+\infty} \lim _{\beta \rightarrow+\infty} \frac{1}{\beta} \log \left(\mu_{\beta}\left(\left[1^{n}\right]\right)\right)=0
\end{aligned}
$$

and, for any $x=\left(x_{1} x_{2} x_{3} \cdots\right), x \notin\left\{0^{\infty}, 1^{\infty}\right\}$,

$$
I(x)=\inf _{y \in\left\{0^{\infty}, 1^{\infty}\right\}} \liminf _{n \rightarrow+\infty}\left(R_{+}^{n}\left(x_{1} \cdots x_{n} y\right)+I(y)\right) .
$$

(It can be checked that Eq. (17) is satisfied for $x=0^{\infty}$ and $x=1^{\infty}$, but this is not necessary).

For any cylinder $k \subset\{0,1\}^{\mathbb{N}}$, we claim that

$$
\lim _{\beta \rightarrow+\infty} \frac{1}{\beta} \log \left(\mu_{\beta}(k)\right)=-\inf _{x \in k} I(x) .
$$

Indeed, for a given cylinder $k_{0}$, from (3), the family $\left(\frac{1}{\beta} \log \left(\mu_{\beta}\left(k_{0}\right)\right)\right)$ is bounded. If for a sequence $\beta_{i} \rightarrow+\infty$, we have that $\frac{1}{\beta_{i}} \log \left(\mu_{\beta_{i}}\left(k_{0}\right)\right)$ converges, then (following the Remark 3., which appears below the Theorem 1) for some subsequence $\beta_{i_{j}}$ of $\beta_{i}$ and for any cylinder $k \subset X$, we have

$$
\lim _{\beta_{i_{j}} \rightarrow+\infty} \frac{1}{\beta_{i_{j}}} \log \left(\mu_{\beta_{i_{j}}}(k)\right)=-\inf _{x \in k} I(x) .
$$

Particularly, we get

$$
\lim _{\beta_{i} \rightarrow+\infty} \frac{1}{\beta_{i}} \log \left(\mu_{\beta_{i}}\left(k_{0}\right)\right)=\lim _{\beta_{i_{j}} \rightarrow+\infty} \frac{1}{\beta_{i_{j}}} \log \left(\mu_{\beta_{i_{j}}}\left(k_{0}\right)\right)=-\inf _{x \in k_{0}} I(x) .
$$


This argument proves that

$$
\lim _{\beta \rightarrow+\infty} \frac{1}{\beta} \log \left(\mu_{\beta}\left(k_{0}\right)\right)=-\inf _{x \in k_{0}} I(x),
$$

which concludes the proof of (18).

Now we study the function $I$. Given a point $x=\left(x_{1} x_{2} x_{3} \ldots\right)$ for which 01 occurs infinitely many times, we have $I(x)=+\infty$ because $I \geq R_{+}^{\infty}$ and for each occurrence of 01 in $x$,

$$
\begin{aligned}
R_{+}\left(01 x_{s} x_{s+1} \cdots\right) & =-b-U\left(01 x_{s} \cdots\right)+U\left(1 x_{s} \cdots\right) \\
& \geq-b-\left(b+d+\sum_{j \geq 2} c_{j}\right)+\left(b+\sum_{j \geq 2} c_{j}\right) \\
& =-b-d>0 .
\end{aligned}
$$

Then, from (9),

$$
I(x)= \begin{cases}R_{+}^{n}(x)+I\left(0^{\infty}\right) & \text { if } x=\left(x_{1} \cdots x_{n} 0^{\infty}\right) \\ R_{+}^{n}(x)+I\left(1^{\infty}\right) & \text { if } x=\left(x_{1} \cdots x_{n} 1^{\infty}\right) . \\ +\infty & \text { else }\end{cases}
$$

Assuming $\sum_{j \geq 2} c_{j}<b+d+\sum_{j \geq 2} a_{j}$ we get a symmetric result.

Theorem 11 Let $f \in \mathbf{W}$ satisfying (11). Suppose $\sum_{j \geq 2} a_{j} \geq b+d+\sum_{j \geq 2} c_{j}$ and $\sum_{j \geq 2} c_{j} \geq b+d+\sum_{j \geq 2} a_{j}$. Then, $\mu_{\beta}$ satisfies a Large Deviation Principle with deviation function $I(x)=R_{+}^{\infty}(x)$. More precisely,

$$
I\left(0^{\infty}\right)=0, \quad I\left(1^{\infty}\right)=0
$$

and for any $x \in\{0,1\}^{\mathbb{N}}$,

$$
I(x)=\left\{\begin{array}{l}
R_{+}^{n}(x) \text { if } x=\left(x_{1} \cdots x_{n} 0^{\infty}\right) \text { or } x=\left(x_{1} \cdots x_{n} 1^{\infty}\right) \\
+\infty \text { else }
\end{array},\right.
$$

where $R_{+}=-f-U+U \circ \sigma$ and $U$ satisfies

$$
\begin{aligned}
& U\left(0^{\infty}\right)=0, \\
& U\left(1^{\infty}\right)=\frac{b}{2}-\frac{d}{2}+\frac{1}{2} \sum_{j \geq 2} a_{j}-\frac{1}{2} \sum_{j \geq 2} c_{j}, \\
& U\left(0^{q} 1 z\right)=\sum_{j \geq 1} a_{q+j},
\end{aligned}
$$




$$
U\left(1^{q} 0 z\right)=\frac{b}{2}-\frac{d}{2}+\frac{1}{2} \sum_{j \geq 2} a_{j}-\frac{1}{2} \sum_{j \geq 2} c_{j}+\sum_{j \geq 1} c_{q+j} .
$$

Remark The above formulas for $U$ can have a more symmetric expression if we add the constant $\frac{d}{2}+\frac{1}{2} \sum_{j \geq 2} c_{j}$. This is irrelevant when we consider the coboundary $U-U \circ \sigma$ in $R_{+}$. Thus we can consider $U$ defined by the formulas

$$
\begin{gathered}
U\left(0^{\infty}\right)=\frac{d}{2}+\frac{1}{2} \sum_{j \geq 2} c_{j}, \quad U\left(1^{\infty}\right)=\frac{b}{2}+\frac{1}{2} \sum_{j \geq 2} a_{j}, \\
U\left(0^{q} 1 z\right)=\frac{d}{2}+\frac{1}{2} \sum_{j \geq 2} c_{j}+\sum_{j \geq 1} a_{q+j}, \quad U\left(1^{q} 0 z\right)=\frac{b}{2}+\frac{1}{2} \sum_{j \geq 2} a_{j}+\sum_{j \geq 1} c_{q+j} .
\end{gathered}
$$

Proof We remark that in the present case (see Lemma 6),

$$
A=\lim _{\beta \rightarrow+\infty} \frac{1}{\beta} \log (P(\beta f))=\frac{b+d}{2}+\sum_{j=1}^{\infty} \frac{a_{1+j}}{2}+\sum_{j=1}^{\infty} \frac{c_{1+j}}{2} .
$$

The proof of this theorem follows the same lines of the above one. We only present some of the steps.

first: $I\left(0^{\infty}\right)=I\left(1^{\infty}\right)=0$. Indeed, as

$$
\sum_{j \geq 2} a_{j} \geq A \geq 2 A \text { and } \sum_{j \geq 2} c_{j} \geq A \geq 2 A,
$$

then, from lemmas 8 and 9 we get, for $\mathrm{n}$ large enough,

$$
\begin{aligned}
\lim _{\beta \rightarrow+\infty} & \frac{1}{\beta} \log \left(\mu_{\beta}\left(\left[0^{n}\right]\right)\right) \\
& =\lim _{\beta \rightarrow+\infty} \frac{1}{\beta} \log \left(S_{0}^{n}(\beta)\right)-\max \left\{\lim _{\beta \rightarrow+\infty} \frac{1}{\beta} \log \left(S_{0}(\beta)\right), \lim _{\beta \rightarrow+\infty} \frac{1}{\beta} \log \left(S_{1}(\beta)\right)\right\} \\
& =-A-\max \{-A,-A\}=0
\end{aligned}
$$

and, similarly,

$$
\lim _{\beta \rightarrow+\infty} \frac{1}{\beta} \log \left(\mu_{\beta}\left(\left[1^{n}\right]\right)\right)=-A-\max \{-A,-A\}=0 .
$$

second: given a point $x=\left(x_{1} x_{2} x_{3} \cdots\right)$ in which 01 occurs infinitely many times, we have $I(x)=+\infty$. Indeed, as in this case we have 10 occurring infinitely many times too, considering $R_{+}^{\infty}(x)$, for each occurrence of 01 or 10 in $x$, we get 


$$
\begin{aligned}
R_{+}\left(01 x_{s} x_{s+1} \cdots\right) & \geq-b-\sum_{j \geq 2} a_{j}+\left(\frac{b}{2}-\frac{d}{2}+\frac{1}{2} \sum_{j \geq 2} a_{j}-\frac{1}{2} \sum_{j \geq 2} c_{j}+\sum_{j \geq 1} c_{1+j}\right) \\
& =\frac{1}{2}\left(\sum_{j \geq 2} c_{j}-b-d-\sum_{j \geq 2} a_{j}\right) \geq 0 ; \\
R_{+}\left(10 x_{s} x_{s+1} \cdots\right) \geq & -d-\left(\frac{b}{2}-\frac{d}{2}+\frac{1}{2} \sum_{j \geq 2} a_{j}+\frac{1}{2} \sum_{j \geq 2} c_{j}\right)+\sum_{j \geq 2} a_{j} \\
& =\frac{1}{2}\left(\sum_{j \geq 2} a_{j}-b-d-\sum_{j \geq 2} c_{j}\right) \geq 0 .
\end{aligned}
$$

This numbers are not zero simultaneously, because their sum results in $-b-d$. Therefore $R_{+}^{\infty}(x)=+\infty$.

From this computations we conclude that the deviation function satisfies,

$$
I(x)= \begin{cases}0 & \text { if } x=0^{\infty} \text { or } x=1^{\infty} \\ R_{+}^{n}(x) & \text { if } x=\left(x_{1} \cdots x_{n} 0^{\infty}\right) \text { or } x=\left(x_{1} \cdots x_{n} 1^{\infty}\right) \\ +\infty & \text { else }\end{cases}
$$

\section{References}

Baraviera, A., Leplaideur, R., Lopes, A.: Ergodic optimization, zero temperature limits and the max-plus algebra. IMPA, Rio de Janeiro (2013)

Baraviera, A., Lopes, A., Mengue, J.: On the selection of subaction and measure for a subclass of potentials defined by P. Walters. Ergodic Theory Dyn. Syst. 33, 1338-1362 (2013)

Baraviera, A., Lopes, A., Thieullen, P.H.: A large deviation principle for equilibrium states of Hölder potencials: the zero temperature case. Stoch. Dyn. 6, 77-96 (2006)

Bissacot, R., Garibaldi, E., Thieullen, P.H.: Zero-temperature phase diagram for double-well type potentials in the summable variation class. Ergodic Theory Dyn. Syst. 1-23 (2016a). doi:10.1017/etds.2016.57

Bissacot, R., Mengue, J., Perez, E.: A large deviation principle for gibbs states on Countable Markov Shifts at Zero Temperature (2016b) (preprint)

Bowen, R.: Equilibrium States and the Ergodic Theory of Anosov Diffeomorphisms. 2nd Edition. Edited by Jean-René Chazottes. Springer-Verlag, Berlin (2008)

Brémont, J.: Gibbs measures at temperature zero. Nonlinearity 16(2), 419-426 (2003)

Chazottes, J.R., Hochman, M.: On the zero-temperature limit of Gibbs states. Comm. Math. Phys 297(1), 265-281 (2010)

Contreras, G., Lopes, A.O.: Lyapunov minimizing measures for expanding maps of the circle. Ergodic Theory Dyn. Syst. 21, 1379-1409 (2001)

Conze, J. P., Guivarc'h, Y.: Croissance des sommes ergodiques et principe variationnel, manuscript circa (1993)

Coronel, D., Rivera-Letelier, J.: Sensitive dependence of Gibbs measures at low temperatures. J. Stat. Phys. 160, 1658-1683 (2015)

Dembo, A., Zeitouni, O.: Large deviation techniques and applications. Springer, Berlin (1998)

Jenkinson, O.: Ergodic optimization. Discrete Contin. Dyn. Syst. Ser. A 15, 197-224 (2006)

Kempton, T.: Zero temperature limits of Gibbs equilibrium states for countable Markov shifts. J. Stat. Phys. 143, 795-806 (2011) 
Leplaideur, R.: A dynamical proof for the convergence of Gibbs measures at temperature zero. Nonlinearity 18, 2847-2880 (2005)

Leplaideur, R.: Flatness is a criterion for selection of maximizing measures. J. Stat. Phys. 147(4), 728-757 (2012)

Lopes, A., Mohr, J., Souza, R.: Negative Entropy, pressure and zero temperature: a LDP for stationary Markov Chains on [0, 1]. Bull. Braz. Math. Soc. 40(1), 1-52 (2009)

Lopes, A., Mengue, J.: Selection of measure and a large deviation principle for the general XY model. Dyn. Syst. 29(1), 24-39 (2014)

Mengue, J.: Zeta-medidas e princípio dos grandes desvios. PhD thesis, UFRGS (2010). http://hdl.handle. net/10183/26002

Morris, I.: A sufficient condition for the subordination principle in ergodic optimization. Bull. Lond. Math. Soc. 39(2), 214-220 (2007)

Morris, I.: Mather sets for sequences of matrices and applications to the study of joint spectral radii. Proc. Lond. Math. Soc. 107, 121-150 (2013)

Parry, W., Pollicott, M.: Zeta functions and the periodic orbit structure of hyperbolic dynamics, Astérisque Vol 187-188 (1990)

Walters, P.: A natural space of functions for the Ruelle operator theorem. Ergodic Theory Dyn. Syst. 27, 1323-1348 (2007) 